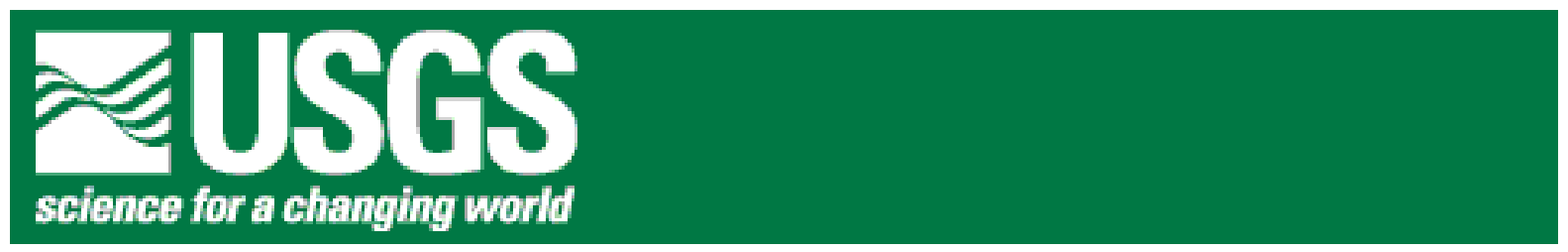

\title{
Results of Chemical and Stable Isotopic Analyses of Water Samples Collected in the Patagonia Mountains, southern Arizona
}

by Richard B. Wanty ${ }^{1}$, Wayne C. Shanks III $^{1}$, Paul Lamothe ${ }^{1}$, Al Meier ${ }^{1}$, Fred Lichte ${ }^{1}$, Paul H. Briggs ${ }^{1}$, and Byron R. Berger ${ }^{1}$

Open-File Report 01-463

2001

This report is preliminary and has not been reviewed for conformity with U.S. Geological Survey editorial standards or with the North American Stratigraphic Code. Any use of trade, firm, or product names is for descriptive purposes only and does not imply endorsement by the U.S. Government. 


\section{U.S. DEPARTMENT OF THE INTERIOR U.S. GEOLOGICAL SURVEY}

${ }^{1}$ Denver, Colorado 
Results of Chemical and Stable Isotopic Analyses of Water Samples Collected in the Patagonia Mountains, southern Arizona

by Richard B. Wanty, Wayne C. Shanks III, Paul Lamothe, Al Meier, Fred Lichte, Paul H. Briggs, and Byron R. Berger

\begin{abstract}
Water samples were collected in the Patagonia Mountains in February, 1997. Most of the samples were collected from portals of abandoned mines, or from stream drainages

immediately downstream from abandoned mines. Most of the samples have low $\mathrm{pH}(<4)$ and high total dissolved solids $(>1000 \mathrm{mg} / \mathrm{L})$. Anion composition of the water samples is dominated by sulfate, while cation compositions range from calcium-dominated to mixed calcium-magnesium or calcium-sodium-dominated waters. Metals such as iron, manganese, copper, zinc, and aluminum contribute a significant portion $(>10 \%)$ of the cation content to the water samples. Because of the low $\mathrm{pH}$ 's, protons contribute up to several percent of the cation character of the waters in some of the samples. The data are presented in tabular and graphical formats, with descriptions of data quality and brief descriptions of results.
\end{abstract}

\title{
Introduction
}

The Patagonia Mountains are located in southern Arizona, between Tucson and Nogales (fig. 1). This area has a history of mining activity that extends back at least 100 years (Schrader, 1915). Primary production has included copper, lead, and zinc, with gold and silver produced from epithermal vein systems. Most of the mines in the area have been inactive and abandoned for many decades, but some continue to produce acidic, metal-rich drainage waters from mine portals or possibly from discharge of ground water that has passed through the mine workings. Drainage from these mines is mostly ephemeral.

The climate in southern Arizona is characterized by semiarid to arid conditions, with monsoon rains producing most of the average annual rainfall during the summer months. Only $25 \%$ of the average annual precipitation is historically experienced during the winter months. 


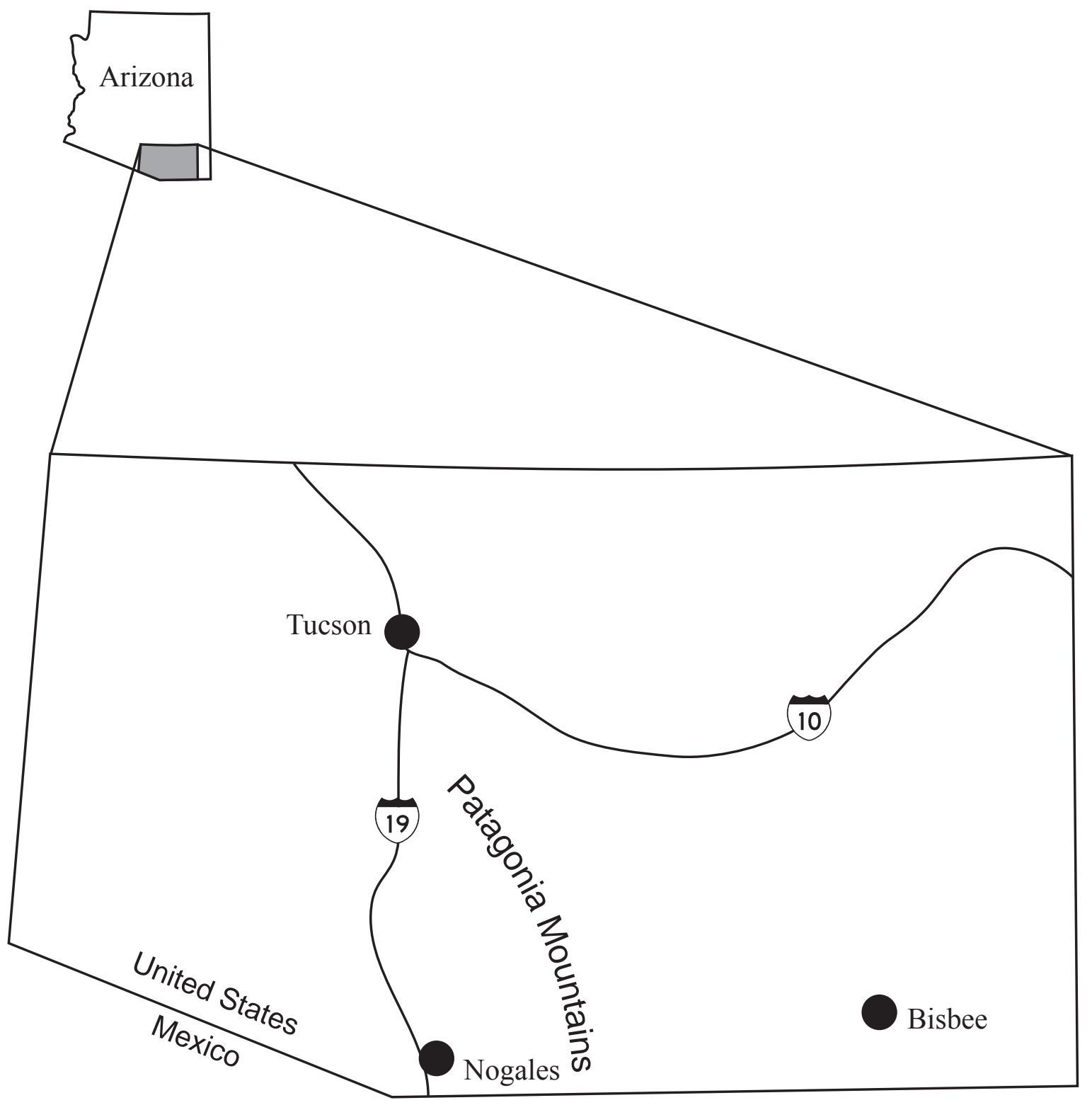

Figure 1- location map for this study. 


\section{Field Methods}

Field sampling was conducted over a period of three days in February, 1997. During that time, air temperatures were $40-50^{\circ} \mathrm{F}$ (approx. $5-15^{\circ} \mathrm{C}$ ). Rain fell during the third day of sampling, at the time that samples 14 through 17 were being collected.

Samples were collected using established methods (cf. Ficklin and Mosier, 1999, for a thorough review). Field analyses were performed for $\mathrm{pH}$, temperature, dissolved oxygen, and electrical conductivity. The $\mathrm{pH}$ measurements were made at each site following a calibration using three known buffer solutions. Because the samples generally had low $\mathrm{pH}$ values, buffers from a similar $\mathrm{pH}$ range were used; usually $\mathrm{pH} 1.68,3.00$ or 4.00, and 7.00 $\left(25^{\circ} \mathrm{C}\right.$ values). The $\mathrm{pH}$ meter had an automatic temperature compensation device so that the $\mathrm{pH}$ calibrations and measurements are corrected for the listed water temperatures.

Temperature was measured using a calibrated digital thermometer. Electrical conductivity was measured as equivalent total dissolved solids expressed as $\mathrm{NaCl}$. At each site except 97AZ-13, a visual estimate of discharge was made; these are approximations only. Sample locations and results of field analyses are shown in Table 1. Other data shown in Table 1 include latitude, longitude, and elevation. These were determined after completion of field work from locations plotted on the USGS 1:24,000 scale topographic sheets for the Cumero Canyon and Harshaw quadrangles. The distribution of sample locations is shown in figure 2.

At each site, samples were collected for later laboratory analyses. These included samples for anions, cations, ferrous iron (for most samples), and for stable isotopes values of

$\delta \mathrm{D}, \delta^{18} \mathrm{O}$, and $\delta^{34} \mathrm{~S}$ in water and in dissolved $\mathrm{SO}_{4}$. The anion samples were filtered through a $0.45 \mu \mathrm{m}$ nominal pore size cellulose acetate filter and stored in low-density polyethylene bottles. Cation samples were similarly filtered and acidified with concentrated Ultrex-grade nitric acid from J.T. Baker Chemical Company (0.5 mL acid per $30 \mathrm{ml} \mathrm{sample})$ and stored in low-density polyethylene bottles. The ferrous iron samples were filtered and acidified with reagent grade hydrochloric acid ( $1 \mathrm{~mL}$ acid per $100 \mathrm{~mL}$ sample) and kept in amber (opaque) high-density polyethylene bottles to avoid photo-oxidation of iron. Anion and ferrous iron samples were kept on ice in a cooler from the time of sampling, then stored in a refrigerator in the laboratory until the time of analysis. 
Table 1- Field analyses, locations and descriptive data for samples collected in the Patagonia Mountains, southern Arizona, in February, 1997. Elevations are given in feet above mean sea level (FAMSL) as determined from 1:24,000-scale topographic maps. Flow estimates were not made for samples 97AZ-13 or 97AZ-17.

\begin{tabular}{|l|l|}
\hline Sample No. & \\
\hline $97 \mathrm{AZ}-1$ & flowing well in Humboldt Canyon \\
\hline $97 \mathrm{AZ}-2$ & Humboldt Canyon, approx. 100m upstream of mine \\
\hline $97 \mathrm{AZ}-3$ & mouth of World's Fair mine \\
\hline $97 \mathrm{AZ}-4$ & World's Fair mine at base of tailings dump \\
\hline $97 \mathrm{AZ}-5$ & 50 m downstream of site $\# 4$ \\
\hline $97 \mathrm{AZ}-6$ & approximately $200 \mathrm{~m}$ downstream of site \#5 \\
\hline $97 \mathrm{AZ}-7$ & approximately $1 \mathrm{~km}$ downstream from W.F. mine in Alum Gulch \\
\hline $97 \mathrm{AZ}-8$ & approximately $500 \mathrm{~m}$ downstream of Three-R mine \\
\hline $97 \mathrm{AZ}-9$ & approximately $800 \mathrm{~m}$ downstream from site \#8 \\
\hline $97 \mathrm{AZ}-10$ & Ventura Mine mouth \\
\hline $97 \mathrm{AZ}-11$ & approx. $150 \mathrm{~m}$ downstream from European Mine \\
\hline $97 \mathrm{AZ}-12$ & flowing well down hill from European Mine \\
\hline $97 \mathrm{AZ}-13$ & Paymaster Spring \\
\hline $97 \mathrm{AZ}-14$ & Alum Gulch approx. 150 m downstream from Humboldt Canyon \\
\hline $97 \mathrm{AZ}-15$ & Same as site \#7 \\
\hline $97 \mathrm{AZ}-16$ & approx. 1 km downstream from site \#15 \\
\hline $97 \mathrm{AZ}-17$ & Alum gulch near Exposed Reef Mine \\
\hline
\end{tabular}

\begin{tabular}{|c|c|c|c|c|c|c|c|}
\hline Sample No. & Latitude & Longitude & $\begin{array}{c}\text { elevation } \\
\text { FAMSL }\end{array}$ & $\begin{array}{c}\text { flow, visual } \\
\text { estimate }\end{array}$ & $\mathrm{pH}$ & $\mathrm{T}^{\circ} \mathrm{C}$ & $\begin{array}{c}\text { TDS, as } \\
\mathrm{NaCl}\end{array}$ \\
\hline $97 \mathrm{AZ}-1$ & 31.4647 & -110.7447 & 5150 & $30 \mathrm{gpm}$ & 3.45 & 19.3 & 160 \\
\hline $97 \mathrm{AZ}-2$ & 31.4686 & -110.7331 & 4860 & $.1-.3 \mathrm{gpm}$ & 2.89 & 9.6 & 650 \\
\hline $97 \mathrm{AZ}-3$ & 31.4797 & -110.7377 & 4800 & $10 \mathrm{gpm}$ & 3.07 & 12.5 & 1300 \\
\hline $97 \mathrm{AZ}-4$ & 31.4802 & -110.7363 & 4650 & $5 \mathrm{gpm}$ & 2.92 & 6.4 & 1300 \\
\hline $97 \mathrm{AZ}-5$ & 31.4812 & -110.7363 & 4640 & $5 \mathrm{gpm}$ & 3.15 & 11.7 & 1860 \\
\hline $97 \mathrm{AZ}-6$ & 31.4831 & -110.7367 & 4575 & $5 \mathrm{gpm}$ & 2.97 & 10.0 & 1820 \\
\hline $97 \mathrm{AZ}-7$ & 31.4857 & -110.7373 & 4500 & $5 \mathrm{gpm}$ & 3.41 & 7.3 & 1750 \\
\hline $97 \mathrm{AZ}-8$ & 31.4767 & -110.7729 & 4520 & $1-2 \mathrm{gpm}$ & 3.12 & 7.2 & 660 \\
\hline $97 \mathrm{AZ}-9$ & 31.4763 & -110.7769 & 4390 & $<.5 \mathrm{gpm}$ & 3.05 & 8.6 & 680 \\
\hline $97 \mathrm{AZ}-10$ & 31.4572 & -110.7640 & 5320 & $.1 \mathrm{gpm}$ & 2.89 & 9.7 & 1620 \\
\hline $97 \mathrm{AZ}-11$ & 31.4648 & -110.7703 & 4800 & $0.02-.04 \mathrm{gpm}$ & 2.88 & 7.6 & 1020 \\
\hline $97 \mathrm{AZ}-12$ & 31.4697 & -110.7813 & 4415 & $.1-.3 \mathrm{gpm}$ & 7.61 & 14.3 & 820 \\
\hline $97 \mathrm{AZ}-13$ & 31.4111 & -110.7052 & 5500 & & 6.86 & 7.3 & 140 \\
\hline $97 \mathrm{AZ}-14$ & 31.4766 & -110.7327 & 4775 & $10-20 \mathrm{gpm}$ & 3.8 & 7.6 & 1770 \\
\hline $97 \mathrm{AZ}-15$ & 31.4857 & -110.7373 & 4500 & $25 \mathrm{gpm}$ & 3.45 & 7.1 & 1750 \\
\hline $97 \mathrm{AZ}-16$ & 31.4895 & -110.7449 & 4390 & $5 \mathrm{gpm}$ & 3.77 & 9.6 & 1710 \\
\hline $97 \mathrm{AZ}-17$ & 31.4937 & -110.7477 & 4350 & & 3.47 & 9.0 & 1510 \\
\hline
\end{tabular}




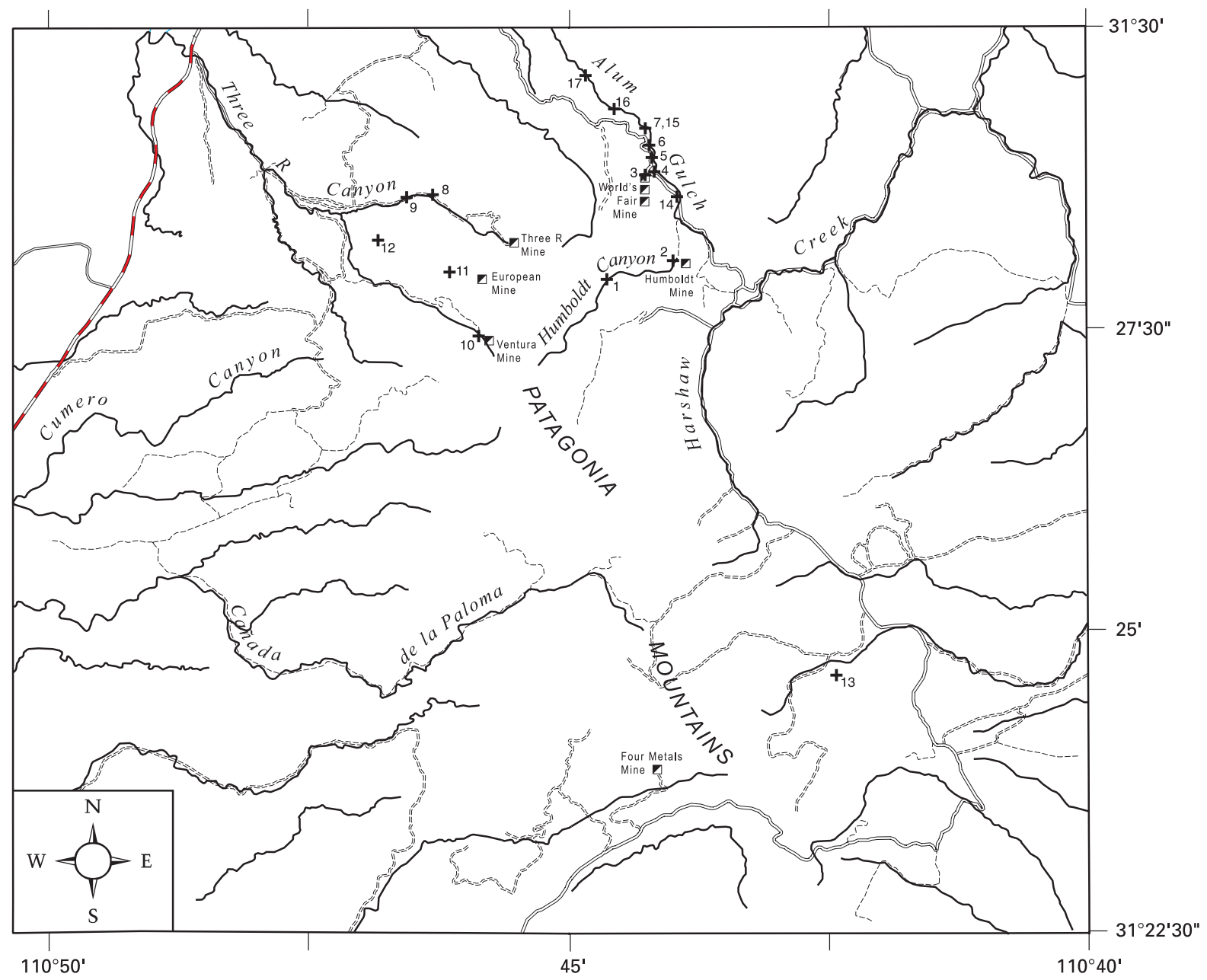

Figure 2- Base map of the Patagonia study area. Sample locations are indicated with a "+," and numbers correspond to sample numbers in the tables, each of which has the prefix, "97AZ-." Surface-water drainages are shown by the thin black lines. Roads are shown with double lines, dashed where not maintained. 


\section{Laboratory Methods}

Ferrous iron determinations were made using Hach Accuvac ${ }^{\circledR}$ ampoules with a Hach DR2000 spectrophotometer. The method involves drawing the sample into an evacuated ampoule that contains a proprietary reagent, then measuring the color development. The method is based on the 1,10 phenanthroline method as outlined in Standard Methods for the Examination of Water and Wastewater (Clesceri and others, 1998).

Anion determinations were made using a Dionex DX-500 ion chromatography (IC) system. Standard running conditions used an AS-4 chromatography column, with a mixed carbonate/bicarbonate eluent. With this method, fluoride and chloride determinations were made. Minor amounts of bromide were detected in most samples, and nitrate was detected in two samples. Sulfate analyses also were performed using the IC method, but the results of sulfate analyses from the ICP-AES are considered to be more reliable because of an analytical problem with the IC system. In the normal sequence of IC analyses, each sample was run, diluted with an equal volume of deionized water. This first sample run provided reliable analytical results for fluoride, chloride, bromide, and nitrate, but sulfate results were always much greater than the linear working range (all samples had $\mathrm{SO}_{4}{ }^{2-}$ concentrations greater than $100 \mathrm{mg} / \mathrm{L}_{\text {as }} \mathrm{SO}_{4}{ }^{2-}$; the instrument is calibrated to $15 \mathrm{mg} / \mathrm{L}$ ). Subsequently a greater dilution was run (usually 1:100, 1:200, or 1:500 v:v) for sulfate. However, a problem was discovered recently wherein there is a carryover effect following the injection of highsulfate samples into the IC, so the sulfate numbers produced by the IC were spuriously high. Thus, the IC sulfate numbers are not reported herein, but rather, sulfate analytical results are reported from the ICP/AES. Further method development work with the IC has shown that dilution of samples with eluent, rather than deionized water, prevents this carryover effect in all but the most sulfate-rich samples.

Cations were determined by inductively coupled plasma / atomic emission spectroscopy (ICP/AES) and inductively coupled plasma / mass spectroscopy (ICP/MS). The ICP/AES method produces preferred values for major cations such as alkali metals and alkaline earths, as well as for some of the trace elements, including iron, aluminum, and manganese. The ICP/MS method has the advantage of greatly increased sensitivity, and is capable of detecting a greater number of elements, so most of the trace element values and rare-earth element analyses were determined by the ICP/MS method (Briggs and Fey, 1996; Lamothe and others, 1999).

Samples for isotopic determinations were placed in $20 \mathrm{~mL}$ glass scintillation vials, tightly capped, and returned to the USGS laboratories in Denver for analyses by isotope ratio mass spectrometry. Water samples were prepared for hydrogen isotopic analyses using the $\mathrm{Zn}$ reduction technique (Coleman et al., 1982) and for oxygen isotope analyses using an automated $\mathrm{CO}_{2}$ equilibration technique. Values of $\delta^{18} \mathrm{O}$ and $\delta \mathrm{D}$ are relative to the VSMOW standard and have reproducibility of approximately 1.0 and $0.7 \%$, respectively. Dissolved sulfate was precipitated in the laboratory as $\mathrm{BaSO}_{4}$, filtered, dried, and reacted with $\mathrm{BrF}_{5}$ to produce $\mathrm{CO}_{2}$ for $\delta^{18} \mathrm{O}$ analysis (Clayton and Mayeda, 1963) on a Finnigan 252 isotope ratio mass spectrometer. $\delta^{18} \mathrm{O}$ error is estimated to be $\pm 1 \%$. A split of each $\mathrm{BaSO}_{4}$ precipitate 
was analyzed for $\delta^{34} \mathrm{~S}$ using an automated elemental analyzer interfaced to a Micromass Optima isotope ratio mass spectrometer. $\delta^{34} \mathrm{~S}$ error is estimated to be $\pm 0.2 \%$.

\section{Results and Discussion}

\section{General results}

Results of field analyses were given in Table 1. Ferrous iron determinations are shown in Table 2 for a subset of the samples collected. Anion results by ion chromatography (except sulfate) are shown in Table 3. Cation analyses were performed by ICP/AES on the same subset of samples for which ferrous analyses were performed. Those results are shown in Table 4. Cation analyses for the full suite of samples are shown in Table 5. Rare earth element analyses for the ferrous-iron subset are shown in Table 6. Results for stable isotopic analyses are shown in Table 7.

\section{Data Quality}

The primary test of the overall quality of the chemical data is indicated by the charge balance calculations, which are shown in Table 8 . In all cases, charge balances are within $\pm 8 \%$. The analyses that contribute to the overall charge balances include, field $(\mathrm{pH})$ and laboratory (anions by IC, cations plus sulfate by ICP/AES) methods. The results of cationanion balances are in excellent agreement, indicating that the analytical results are reliable.

A second test of data quality is found in running field and laboratory blanks, using deionized or distilled water. A field blank was prepared using distilled water that was carried to a field site and treated as though it were a sample. Field-blank samples were run for anions (by IC) and cations (by ICP/MS) and in both cases, showed below-detection values for all components. This result demonstrates that the likelihood of sample contamination is very low.

A third test of data quality is given by running standards as though they are samples, and comparing the analytical results with most probable values given for the standards. For the IC, ICP/MS, and ICP/AES, at least $10 \%$ of the analyses were performed on standards run as unknowns. This test allows a check of analytical precision and accuracy through the data set. In addition, a minimum of $10 \%$ sample duplicates was run for the IC and ICP/AES as a further check of analytical precision. 
Table 2- Summary of analytical results for ferrous and total iron. Sampling, preservation, and analytical methods are described above. All concentrations are given in milligrams per liter $(\mathrm{mg} / \mathrm{L})$. The $\mathrm{Fe}^{2+} / \mathrm{Fe}^{\text {tot }}$ ratio is dimensionless.

\begin{tabular}{|c|c|c|c|}
\hline & $\mathrm{Fe}^{2+}$ & total Fe & $\mathrm{Fe}^{2+} / \mathrm{Fe}^{\text {tot }}$ \\
\hline & (colorimetric) & (ICP/AES) & \\
\hline 97AZ-1 & 0.26 & 0.74 & $35 \%$ \\
\hline 97AZ-2 & 0.18 & 3.1 & $5.8 \%$ \\
\hline 97AZ-3 & 130 & 130 & $99 \%$ \\
\hline 97AZ-4 & 21 & 93 & $22 \%$ \\
\hline 97AZ-5 & 0.94 & 11 & $8.5 \%$ \\
\hline 97AZ-6 & 0.31 & 6.0 & $5.2 \%$ \\
\hline 97AZ-7 & 0.38 & 1.0 & $38 \%$ \\
\hline 97AZ-8 & 0.02 & 1.5 & $1.3 \%$ \\
\hline 97AZ-10 & 210 & 240 & $88 \%$ \\
\hline 97AZ-11 & 1.5 & 47 & $3.2 \%$ \\
\hline 97AZ-17 & 0.67 & 1.3 & $54 \%$ \\
\hline
\end{tabular}


Table 3- Results of anion analyses by ion chromatography (IC). Sampling, preservation, and analytical methods are described in the text. Nitrate is give as milligrams per liter as $\mathrm{NO}_{3}$. To convert to milligrams per liter as $\mathrm{N}$, multiply the result given by 0.23 . A dash indicates no detectable quantity.

\begin{tabular}{|c|c|c|c|c|}
\hline Sample & $\mathrm{F}$ & $\mathrm{Cl}$ & $\mathrm{Br}$ & $\mathrm{NO}_{3}$ \\
\hline Number & $\mathrm{mg} / \mathrm{L}$ & $\mathrm{mg} / \mathrm{L}$ & $\mathrm{mg} / \mathrm{L}$ & $\mathrm{mg} / \mathrm{L}_{\text {as }} \mathrm{NO}_{3}$ \\
\hline $97 A Z-1$ & 0.35 & 4.7 & - & - \\
\hline $97 A Z-2$ & 0.15 & 9.9 & 0.4 & - \\
\hline $97 A Z-3$ & 1.8 & 10. & - & - \\
\hline $97 A Z-4$ & 2.0 & 13 & - & - \\
\hline $97 A Z-5$ & 0.95 & 16. & 0.2 & - \\
\hline $97 A Z-6$ & 0.85 & 16. & 0.1 & - \\
\hline $97 A Z-7$ & 1.1 & 17. & 0.2 & - \\
\hline $97 A Z-8$ & 0.2 & 9.9 & 0.7 & - \\
\hline $97 A Z-9$ & 0.33 & 13. & 0.68 & 2.2 \\
\hline $97 A Z-10$ & 0.33 & 22. & 0.5 & - \\
\hline $97 A Z-11$ & 0.6 & 14. & 0.3 & - \\
\hline $97 A Z-12$ & 0.61 & 7.4 & - & - \\
\hline $97 A Z-13$ & 0.21 & 5 & - & - \\
\hline $97 A Z-14$ & 0.83 & 17 & 0.6 & 0.6 \\
\hline $97 A Z-15$ & 1 & 17 & 0.1 & - \\
\hline $97 A Z-16$ & 2.1 & 17 & 0.1 & - \\
\hline $97 A Z-17$ & 2.2 & 18. & 0.2 & - \\
\hline
\end{tabular}


Table 4- Results of cation analyses by ICP/AES. Values are given in the units indicated in each column. The following elements were reported as being below the lower limit of detection for all samples: $\mathrm{Ag}, \mathrm{Cr}(<10 \mu \mathrm{g} / \mathrm{L})$; $\mathrm{Mo}(<20 \mu \mathrm{g} / \mathrm{L})$; and As, $\mathrm{P}$, and $\mathrm{Sb}($ all $<100$ $\mu \mathrm{g} / \mathrm{L})$.

\begin{tabular}{|c|c|c|c|c|c|c|c|}
\hline Field No & $\mathrm{Ca} \mathrm{mg} / \mathrm{L}$ & $\mathrm{Mg} \mathrm{mg} / \mathrm{L}$ & $\mathrm{Na} \mathrm{mg} / \mathrm{L}$ & $\mathrm{K} \mathrm{mg} / \mathrm{L}$ & $\mathrm{SO}_{4} \mathrm{mg} / \mathrm{L}$ & $\mathrm{Si} \mathrm{mg/L}$ & $\mathrm{Fe} \mathrm{mg/L}$ \\
\hline 97AZ-1 & 5.7 & 3.0 & 4.3 & 1.9 & 110 & 13. & 0.74 \\
\hline 97AZ-2 & 12. & 9.8 & 8.5 & 3.0 & 680 & 29. & 3.1 \\
\hline 97AZ-3 & 170 & 100 & 11. & 27. & 1700 & 40. & 140 \\
\hline 97AZ-4 & 180 & 110 & 12. & 24. & 1700 & 40. & 93. \\
\hline $97 \mathrm{AZ}-5$ & 380 & 210 & 40. & 7.3 & 2500 & 35. & 11. \\
\hline 97AZ-6 & 390 & 210 & 40. & 6.3 & 2500 & 35. & 6.0 \\
\hline $97 \mathrm{AZ}-7$ & 400 & 200 & 41. & 5.1 & 2500 & 34. & 1.0 \\
\hline 97AZ-8 & 15 & 8.5 & 22. & 11. & 730 & 49. & 1.5 \\
\hline 97AZ-10 & 260 & 130 & 60. & 7.2 & 2500 & 37. & 240 \\
\hline 97AZ-11 & 90. & 48. & 36. & 3.5 & 1200 & 51. & 47. \\
\hline 97AZ-17 & 420 & 180 & 48. & 4.4 & 2500 & 31. & 1.3 \\
\hline
\end{tabular}

\begin{tabular}{|c|c|c|c|c|c|c|c|}
\hline Field No & $\mathrm{Li} \mu \mathrm{g} / \mathrm{L}$ & $\mathrm{Be} \mu \mathrm{g} / \mathrm{L}$ & $\mathrm{B} \mu \mathrm{g} / \mathrm{L}$ & $\mathrm{Al} \mathrm{mg} / \mathrm{L}$ & $\mathrm{V} \mu \mathrm{g} / \mathrm{L}$ & $\mathrm{Mn} \mathrm{mg} / \mathrm{L}$ & $\mathrm{Co} \mu \mathrm{g} / \mathrm{L}$ \\
\hline 97AZ-1 & $<10$ & $<10$ & 18. & 12. & $<10$ & .33 & 26. \\
\hline 97AZ-2 & 16. & $<10$ & 15. & 98. & $<10$ & 5.2 & 130 \\
\hline 97AZ-3 & 110 & 19. & 24. & 50. & $<10$ & 150 & 360 \\
\hline 97AZ-4 & 101 & 19. & 29. & 52. & $<10$ & 160 & 360 \\
\hline 97AZ-5 & 120 & 12. & 31. & 75. & $<10$ & 140 & 260 \\
\hline 97AZ-6 & 120 & 15. & 27. & 73. & $<10$ & 140 & 280 \\
\hline 97AZ-7 & 110 & 14. & 24. & 73. & $<10$ & 140. & 270 \\
\hline 97AZ-8 & 37. & $<10$ & 16. & 83. & $<10$ & 2.0 & 180 \\
\hline 97AZ-10 & 88. & 15. & 21. & 130 & 23. & 41. & 430 \\
\hline 97AZ-11 & 56. & $<10$ & 16. & 84. & $<10$ & 19. & 240 \\
\hline 97AZ-17 & 110 & 15. & 22. & 86. & $<10$ & 110 & 280 \\
\hline
\end{tabular}

\begin{tabular}{|c|c|c|c|c|c|c|c|}
\hline Field No & $\mathrm{Ni} \mu \mathrm{g} / \mathrm{L}$ & $\mathrm{Cu} \mu \mathrm{g} / \mathrm{L}$ & $\mathrm{Zn} \mu \mathrm{g} / \mathrm{L}$ & $\mathrm{Sr} \mu \mathrm{g} / \mathrm{L}$ & $\mathrm{Cd} \mu \mathrm{g} / \mathrm{L}$ & $\mathrm{Ba} \mu \mathrm{g} / \mathrm{L}$ & $\mathrm{Pb} \mu \mathrm{g} / \mathrm{L}$ \\
\hline 97AZ-1 & 22. & 510 & 410 & 24. & $<5$ & 24. & $<50$ \\
\hline 97AZ-2 & 110 & 4,200 & 7,400 & 59. & 52. & 18. & $<50$ \\
\hline 97AZ-3 & 310 & 96. & 48,000 & 42. & 29. & 12. & $<50$ \\
\hline 97AZ-4 & 320 & 110 & 49,000 & 44. & 29. & 11. & $<50$ \\
\hline 97AZ-5 & 380 & 2,300 & 69,000 & 430 & 220 & 7.2 & $<50$ \\
\hline 97AZ-6 & 370 & 2,300 & 70,000 & 440 & 220 & 14. & 170 \\
\hline 97AZ-7 & 360 & 2,600 & 68,000 & 460 & 230 & 11. & 250 \\
\hline 97AZ-8 & 91. & 77,000 & 2,900 & 61. & 130 & 16. & 67. \\
\hline 97AZ-10 & 290 & 21,000 & 18,000 & 93. & 62. & 9.3 & $<50$ \\
\hline 97AZ-11 & 58. & 12,000 & 8,000 & 85. & 39. & 9.5 & $<50$ \\
\hline 97AZ-17 & 360 & 2,600 & 59,000 & 550 & 220 & 13. & $<50$ \\
\hline
\end{tabular}


Table 5- Results of cation analyses by ICP/MS. All values are shown in $\mu \mathrm{g} / \mathrm{L}$. The following results are not shown: $\mathrm{Au}($ all $<0.01 \mu \mathrm{g} / \mathrm{L}) ; \mathrm{Bi}($ all $<0.05 \mu \mathrm{g} / \mathrm{L}) ; \mathrm{Nb}($ all $<0.1 \mu \mathrm{g} / \mathrm{L})$; Ge (all $<0.2 \mu \mathrm{g} / 1$, except 97AZ-12, which was $1 \mu \mathrm{g} / \mathrm{L}) ; \mathrm{Sn}($ all $<1 \mu \mathrm{g} / \mathrm{L}) ; \mathrm{Se}($ all $<5 \mu \mathrm{g} / \mathrm{L})$; Sc $($ all $<10 \mu \mathrm{g} / \mathrm{L}) ; \mathrm{P}($ all $<100 \mu \mathrm{g} / \mathrm{L})$;

\begin{tabular}{|c|c|c|c|c|c|c|c|}
\hline Sample Number & $\mathrm{Ca}$ & $\mathrm{Mg}$ & $\mathrm{Na}$ & $\mathrm{K}$ & $\mathrm{SiO}_{2}$ & $\mathrm{Fe}$ & $\mathrm{Mn}$ \\
\hline detection limit & 100 & 5. & 100 & 100 & 500 & 500 & 10. \\
\hline $97 \mathrm{AZ}-1$ & 4,500 & 3,000 & 3,600 & 1,900 & 16,000 & 250 & 280 \\
\hline $97 \mathrm{AZ}-2$ & 9,500 & 9,100 & 7,300 & 3,000 & 34,000 & 2,300 & 4500 \\
\hline $97 \mathrm{AZ}-3$ & 150,000 & 77,000 & 8,700 & 26,000 & 43,000 & 110,000 & 140,000 \\
\hline $97 \mathrm{AZ}-4$ & 150,000 & 80,000 & 9,000 & 24,000 & 44,000 & 78,000 & 150,000 \\
\hline $97 \mathrm{AZ}-5$ & 370,000 & 170,000 & 32,000 & 7,300 & 38,000 & 9,500 & 130,000 \\
\hline $97 \mathrm{AZ}-6$ & 380,000 & 170,000 & 32,000 & 6,400 & 39,000 & 4,900 & 130,000 \\
\hline $97 \mathrm{AZ}-7$ & 390,000 & 170,000 & 35,000 & 5,500 & 39,000 & 870 & 130,000 \\
\hline $97 \mathrm{AZ}-8$ & 11,000 & 7,100 & 18,000 & 11,000 & 56,000 & 900 & 1,300 \\
\hline $97 \mathrm{AZ}-9$ & 14,000 & 11,000 & 24,000 & 12,000 & 57,000 & 700 & 2400 \\
\hline $97 \mathrm{AZ}-10$ & 240,000 & 100,000 & 48,000 & 7,200 & 39,000 & 210,000 & 37,000 \\
\hline $97 \mathrm{AZ}-11$ & 77,000 & 38,000 & 31,000 & 3,600 & 64,000 & 40,000 & 17,000 \\
\hline $97 \mathrm{AZ}-12$ & 210,000 & 36,000 & 100,000 & 12,000 & 8,000 & 350 & 380 \\
\hline $97 \mathrm{AZ}-13$ & 28,000 & 4,500 & 15,000 & 4,000 & 29,000 & 80 & 34 \\
\hline $97 \mathrm{AZ}-14$ & 430,000 & 200,000 & 40,000 & 5,800 & 32,000 & 160 & 110,000 \\
\hline $97 \mathrm{AZ}-15$ & 400,000 & 170,000 & 35,000 & 5,500 & 39,000 & 930 & 130,000 \\
\hline $97 \mathrm{AZ}-16$ & 430,000 & 150,000 & 35,000 & 4,100 & 35,000 & 430 & 110,000 \\
\hline $97 \mathrm{AZ}-17$ & 420,000 & 150,000 & 39,000 & 4,500 & 34,000 & 860 & 100,000 \\
\hline
\end{tabular}

\begin{tabular}{|c|c|c|c|c|c|c|c|}
\hline Sample Number & $\mathrm{Li}$ & $\mathrm{Be}$ & $\mathrm{Al}$ & $\mathrm{Ti}$ & $\mathrm{V}$ & $\mathrm{Cr}$ & $\mathrm{Co}$ \\
\hline detection limit & 0.30 & 0.20 & 1.0 & 2. & 10. & 10. & 0.50 \\
\hline $97 \mathrm{AZ}-1$ & 4 & $<0.2$ & 9,800 & $<2$ & $<10$ & $<10$ & 21 \\
\hline $97 \mathrm{AZ}-2$ & 16 & 3 & 81,000 & 3 & $<10$ & $<10$ & 100 \\
\hline $97 \mathrm{AZ}-3$ & 99 & 17 & 37,000 & $<2$ & $<10$ & 10 & 280 \\
\hline $97 \mathrm{AZ}-4$ & 100 & 18 & 39,000 & $<2$ & $<10$ & $<10$ & 290 \\
\hline $97 \mathrm{AZ}-5$ & 120 & 11 & 57,000 & $<2$ & $<10$ & $<10$ & 230 \\
\hline $97 \mathrm{AZ}-6$ & 120 & 15 & 56,000 & 2 & $<10$ & $<10$ & 240 \\
\hline $97 \mathrm{AZ}-7$ & 120 & 14 & 57,000 & $<2$ & $<10$ & $<10$ & 240 \\
\hline $97 \mathrm{AZ}-8$ & 38 & 3 & 64,000 & $<2$ & $<10$ & $<10$ & 130 \\
\hline $97 \mathrm{AZ}-9$ & 50 & 6 & 60,000 & $<2$ & $<10$ & $<10$ & 110 \\
\hline $97 \mathrm{AZ}-10$ & 88 & 14 & 97,000 & $<2$ & 24 & 15 & 330 \\
\hline $97 \mathrm{AZ}-11$ & 59 & 9 & 69,000 & 2 & $<10$ & $<10$ & 200 \\
\hline $97 \mathrm{AZ}-12$ & 260 & $<0.2$ & 3 & 2 & $<10$ & 11 & $<0.5$ \\
\hline $97 \mathrm{AZ}-13$ & 53 & $<0.2$ & 17 & 2 & $<10$ & 10 & $<0.5$ \\
\hline $97 \mathrm{AZ}-14$ & 99 & 4 & 36,000 & $<2$ & $<10$ & $<10$ & 130 \\
\hline $97 \mathrm{AZ}-15$ & 120 & 14 & 58,000 & 2 & $<10$ & $<10$ & 240 \\
\hline $97 \mathrm{AZ}-16$ & 100 & 14 & 60,000 & $<2$ & $<10$ & $<10$ & 230 \\
\hline $97 \mathrm{AZ}-17$ & 98 & 14 & 66,000 & $<2$ & $<10$ & $<10$ & 240 \\
\hline
\end{tabular}




\begin{tabular}{|c|c|c|c|c|c|c|c|}
\hline Sample Number & $\mathrm{Ni}$ & $\mathrm{Cu}$ & $\mathrm{Zn}$ & $\mathrm{Ga}$ & $\mathrm{As}$ & $\mathrm{Rb}$ & $\mathrm{Sr}$ \\
\hline detection limit & 1.0 & 0.50 & 0.50 & 0.50 & 0.50 & 0.10 & 0.10 \\
\hline $97 \mathrm{AZ}-1$ & 19 & 450 & 350 & $<0.5$ & 2.1 & 10 & 20 \\
\hline $97 \mathrm{AZ}-2$ & 91 & 3,800 & 5,700 & 1 & $<0.5$ & 16 & 55 \\
\hline $97 \mathrm{AZ}-3$ & 240 & 100 & 39,000 & 1 & 0.5 & 170 & 42 \\
\hline $97 \mathrm{AZ}-4$ & 240 & 120 & 41,000 & 1 & 0.6 & 180 & 43 \\
\hline $97 \mathrm{AZ}-5$ & 290 & 1,900 & 57,000 & 1 & 6.5 & 44 & 430 \\
\hline $97 \mathrm{AZ}-6$ & 290 & 1,900 & 58,000 & 1 & 0.8 & 36 & 450 \\
\hline $97 \mathrm{AZ}-7$ & 290 & 2,300 & 58,000 & 1 & $<0.5$ & 21 & 500 \\
\hline $97 \mathrm{AZ}-8$ & 63 & 140,000 & 1,600 & $<0.5$ & $<0.5$ & 44 & 52 \\
\hline $97 \mathrm{AZ}-9$ & 69 & 90,000 & 1,700 & $<0.5$ & $<0.5$ & 58 & 49 \\
\hline $97 \mathrm{AZ}-10$ & 230 & 21,000 & 13,000 & 3 & 1.6 & 87 & 91 \\
\hline $97 \mathrm{AZ}-11$ & 41 & 12,000 & 6,100 & 1 & 0.7 & 40 & 81 \\
\hline $97 \mathrm{AZ}-12$ & $<1$ & 1 & 2 & $<0.5$ & 61. & 43 & 650 \\
\hline $97 \mathrm{AZ}-13$ & 1 & 2 & 20 & $<0.5$ & 8.9 & 7 & 94 \\
\hline $97 \mathrm{AZ}-14$ & 260 & 1,800 & 58,000 & 1 & $<0.5$ & 18 & 640 \\
\hline $97 \mathrm{AZ}-15$ & 300 & 2,300 & 59,000 & 1 & $<0.5$ & 20 & 500 \\
\hline $97 \mathrm{AZ}-16$ & 270 & 2,100 & 53,000 & 1 & $<0.5$ & 11 & 600 \\
\hline $97 \mathrm{AZ}-17$ & 280 & 2,100 & 50,000 & 1 & $<0.5$ & 12 & 580 \\
\hline
\end{tabular}

\begin{tabular}{|c|c|c|c|c|c|c|c|}
\hline Sample Number & $\mathrm{Zr}$ & $\mathrm{Mo}$ & $\mathrm{Ag}$ & $\mathrm{Cd}$ & $\mathrm{In}$ & $\mathrm{Sb}$ & $\mathrm{Te}$ \\
\hline detection limit & 0.10 & 0.10 & 0.05 & 0.10 & 0.05 & 0.05 & 0.5 \\
\hline 97AZ-1 & $<0.10$ & $<0.10$ & 0.05 & 2.8 & $<0.05$ & 0.12 & $<0.5$ \\
\hline $97 \mathrm{AZ}-2$ & 0.9 & $<0.10$ & $<0.05$ & 47. & 0.33 & 0.22 & $<0.5$ \\
\hline $97 \mathrm{AZ}-3$ & $<0.10$ & $<0.10$ & $<0.05$ & 28. & $<0.05$ & 0.58 & $<0.5$ \\
\hline $97 \mathrm{AZ}-4$ & $<0.10$ & $<0.10$ & $<0.05$ & 30. & $<0.05$ & 0.36 & $<0.5$ \\
\hline $97 \mathrm{AZ}-5$ & 0.1 & $<0.10$ & 0.11 & 210 & 0.06 & 1.5 & $<0.5$ \\
\hline $97 \mathrm{AZ}-6$ & 0.1 & $<0.10$ & 0.35 & 220 & 0.08 & 1.7 & $<0.5$ \\
\hline $97 \mathrm{AZ}-7$ & 0.1 & $<0.10$ & 0.25 & 220 & 0.09 & 1.4 & $<0.5$ \\
\hline $97 \mathrm{AZ}-8$ & 1.2 & $<0.10$ & $<0.05$ & 110 & 0.09 & 0.13 & $<0.5$ \\
\hline $97 \mathrm{AZ}-9$ & 0.6 & $<0.10$ & $<0.05$ & 52. & 0.07 & 0.08 & $<0.5$ \\
\hline $97 \mathrm{AZ}-10$ & 0.2 & $<0.10$ & 0.05 & 60. & 0.45 & 0.40 & 2.0 \\
\hline $97 \mathrm{AZ}-11$ & 0.3 & $<0.10$ & $<0.05$ & 37. & 0.18 & 0.16 & $<0.5$ \\
\hline $97 \mathrm{AZ}-12$ & $<0.10$ & 2.4 & $<0.05$ & $<0.1$ & $<0.05$ & 0.86 & $<0.5$ \\
\hline $97 \mathrm{AZ}-13$ & $<0.10$ & 0.3 & 0.12 & 0.2 & $<0.05$ & 0.38 & $<0.5$ \\
\hline $97 \mathrm{AZ}-14$ & 0.1 & $<0.10$ & 0.10 & 220 & $<0.05$ & 0.25 & $<0.5$ \\
\hline $97 \mathrm{AZ}-15$ & 0.1 & $<0.10$ & 0.21 & 230 & 0.10 & 1.4 & $<0.5$ \\
\hline $97 \mathrm{AZ}-16$ & 0.2 & $<0.10$ & 0.18 & 220 & $<0.05$ & 0.30 & $<0.5$ \\
\hline $97 \mathrm{AZ}-17$ & 0.2 & $<0.10$ & 0.15 & 210 & $<0.05$ & 0.30 & $<0.5$ \\
\hline
\end{tabular}




\begin{tabular}{|c|c|c|c|c|c|c|c|}
\hline Sample Number & $\mathrm{Cs}$ & $\mathrm{Ba}$ & $\mathrm{Ta}$ & $\mathrm{W}$ & $\mathrm{Re}$ & $\mathrm{Tl}$ & $\mathrm{Pb}$ \\
\hline detection limit & 0.05 & 0.05 & 0.05 & 0.05 & 0.05 & 0.10 & 0.05 \\
\hline 97AZ-1 & 0.34 & 9.1 & $<0.05$ & $<0.05$ & 0.29 & 0.39 & 2.1 \\
\hline $97 \mathrm{AZ}-2$ & 0.36 & 6.4 & 0.09 & $<0.05$ & 0.72 & 0.19 & 0.26 \\
\hline $97 \mathrm{AZ}-3$ & 7.7 & 0.63 & 0.22 & $<0.05$ & 0.34 & 0.46 & 8.7 \\
\hline $97 \mathrm{AZ}-4$ & 8.3 & 0.45 & 0.21 & $<0.05$ & 0.34 & 0.46 & 14. \\
\hline $97 \mathrm{AZ}-5$ & 1.5 & 1.2 & 0.34 & $<0.05$ & 0.53 & 0.25 & 42. \\
\hline $97 \mathrm{AZ}-6$ & 1.1 & 1.9 & 0.22 & $<0.05$ & 0.54 & 0.24 & 210 \\
\hline $97 \mathrm{AZ}-7$ & 0.41 & 4.3 & 0.30 & $<0.05$ & 0.55 & 0.20 & 270 \\
\hline $97 \mathrm{AZ}-8$ & 2.2 & 5.6 & 0.09 & 0.18 & 1.9 & 0.44 & 0.47 \\
\hline $97 \mathrm{AZ}-9$ & 3.0 & 4.5 & 0.07 & 0.06 & 0.93 & 0.58 & 0.32 \\
\hline $97 \mathrm{AZ}-10$ & 6.9 & 0.06 & 0.43 & $<0.05$ & 1.1 & 1.9 & 0.40 \\
\hline $97 \mathrm{AZ}-11$ & 3.4 & 0.21 & 0.14 & $<0.05$ & 0.19 & 0.51 & 1.2 \\
\hline $97 \mathrm{AZ}-12$ & 24. & 7.8 & 0.09 & 30. & $<0.05$ & $<0.1$ & 0.17 \\
\hline $97 \mathrm{AZ}-13$ & 1.2 & 36. & $<0.05$ & $<0.05$ & $<0.05$ & $<0.1$ & 0.49 \\
\hline $97 \mathrm{AZ}-14$ & 0.13 & 5.1 & 0.15 & $<0.05$ & 0.49 & 0.14 & 61. \\
\hline $97 \mathrm{AZ}-15$ & 0.39 & 4.1 & 0.20 & $<0.05$ & 0.58 & 0.22 & 260 \\
\hline $97 \mathrm{AZ}-16$ & 1.1 & 5.0 & 0.17 & $<0.05$ & 0.69 & $<0.1$ & 16. \\
\hline $97 \mathrm{AZ}-17$ & 0.60 & 4.4 & 0.15 & $<0.05$ & 0.64 & 0.11 & 18. \\
\hline
\end{tabular}

\begin{tabular}{|c|c|c|}
\hline Sample Number & Th & $\mathrm{U}$ \\
\hline detection limit & 0.05 & 0.02 \\
\hline 97AZ-1 & $<0.05$ & 2.0 \\
\hline 97AZ-2 & 1.9 & 10. \\
\hline $97 \mathrm{AZ}-3$ & 0.09 & 8.6 \\
\hline $97 \mathrm{AZ}-4$ & 0.10 & 8.9 \\
\hline $97 \mathrm{AZ}-5$ & 0.10 & 11. \\
\hline $97 \mathrm{AZ}-6$ & 0.21 & 12. \\
\hline $97 \mathrm{AZ}-7$ & 0.15 & 11. \\
\hline $97 \mathrm{AZ}-8$ & 30. & 69. \\
\hline $97 \mathrm{AZ}-9$ & 13. & 59. \\
\hline $97 \mathrm{AZ}-10$ & 6.7 & 67. \\
\hline $97 \mathrm{AZ}-11$ & 7.6 & 52. \\
\hline $97 \mathrm{AZ}-12$ & $<0.05$ & 3.9 \\
\hline $97 \mathrm{AZ}-13$ & $<0.05$ & 0.13 \\
\hline $97 \mathrm{AZ}-14$ & 0.05 & 3.6 \\
\hline $97 \mathrm{AZ}-15$ & 0.14 & 11. \\
\hline $97 \mathrm{AZ}-16$ & 0.08 & 9.4 \\
\hline $97 \mathrm{AZ}-17$ & 0.11 & 8.7 \\
\hline
\end{tabular}


Table 6- Results of analyses of rare earth elements and yttrium, lanthanum, and hafnium by ICP/MS. All values are given in $\mu \mathrm{g} / \mathrm{L}$.

\begin{tabular}{|c|c|c|c|c|c|c|c|c|}
\hline Sample Number & $\mathrm{Y}$ & $\mathrm{La}$ & $\mathrm{Ce}$ & $\mathrm{Pr}$ & $\mathrm{Nd}$ & $\mathrm{Sm}$ & $\mathrm{Eu}$ & $\mathrm{Gd}$ \\
\hline detection limit & 0.10 & 0.05 & 0.05 & 0.05 & 0.05 & 0.05 & 0.05 & 0.05 \\
\hline $97 \mathrm{AZ}-1$ & 4.9 & 0.26 & 1.4 & 0.34 & 1.8 & 0.46 & 0.13 & 0.62 \\
\hline $97 \mathrm{AZ}-2$ & 24. & 9.3 & 26. & 3.7 & 16. & 3.6 & 1.0 & 4.1 \\
\hline $97 \mathrm{AZ}-3$ & 120 & 13. & 59. & 13. & 71. & 21. & 5.8 & 26. \\
\hline $97 \mathrm{AZ}-4$ & 120 & 14. & 61. & 14. & 73. & 21. & 6.2 & 27. \\
\hline $97 \mathrm{AZ}-5$ & 150 & 40. & 130 & 22. & 110 & 27. & 7.4 & 32. \\
\hline $97 \mathrm{AZ}-6$ & 150 & 42. & 130 & 22. & 110 & 27. & 7.4 & 31. \\
\hline $97 \mathrm{AZ}-7$ & 150 & 49. & 140 & 23. & 110 & 27. & 7.3 & 31. \\
\hline $97 \mathrm{AZ}-8$ & 51. & 32. & 79. & 10. & 39. & 9.3 & 1.1 & 9.6 \\
\hline $97 \mathrm{AZ}-9$ & 67. & 32. & 85. & 12. & 47. & 12. & 1.4 & 13. \\
\hline $97 \mathrm{AZ}-10$ & 190 & 17. & 72. & 15. & 79. & 23. & 5.8 & 30. \\
\hline $97 \mathrm{AZ}-11$ & 130 & 34. & 100 & 16. & 69. & 18. & 3.0 & 20. \\
\hline $97 \mathrm{AZ}-12$ & $<0.1$ & $<0.05$ & $<0.05$ & $<0.05$ & $<0.05$ & $<0.05$ & $<0.05$ & $<0.05$ \\
\hline $97 \mathrm{AZ}-13$ & $<0.1$ & $<0.05$ & $<0.05$ & $<0.05$ & $<0.05$ & $<0.05$ & $<0.05$ & $<0.05$ \\
\hline $97 \mathrm{AZ}-14$ & 99. & 32. & 81. & 12. & 52. & 13. & 3.5 & 16. \\
\hline $97 \mathrm{AZ}-15$ & 160 & 48. & 140. & 23. & 110 & 27. & 7.3 & 31. \\
\hline $97 \mathrm{AZ}-16$ & 190 & 99. & 260. & 37. & 160 & 37. & 9.5 & 40. \\
\hline $97 \mathrm{AZ}-17$ & 200 & 96 & 260. & 37. & 160 & 38. & 9.7 & 41. \\
\hline
\end{tabular}

\begin{tabular}{|c|c|c|c|c|c|c|c|c|}
\hline Sample Number & $\mathrm{Tb}$ & $\mathrm{Dy}$ & $\mathrm{Ho}$ & $\mathrm{Er}$ & $\mathrm{Tm}$ & $\mathrm{Yb}$ & $\mathrm{Lu}$ & $\mathrm{Hf}$ \\
\hline detection limit & 0.05 & 0.05 & 0.05 & 0.05 & 0.05 & 0.05 & 0.05 & 0.05 \\
\hline 97AZ-1 & 0.13 & 0.87 & 0.16 & 0.50 & 0.06 & 0.41 & 0.06 & $<0.05$ \\
\hline $97 \mathrm{AZ}-2$ & 0.69 & 4.3 & 0.76 & 2.1 & 0.30 & 1.8 & 0.25 & $<0.05$ \\
\hline $97 \mathrm{AZ}-3$ & 3.9 & 21. & 3.9 & 10. & 1.3 & 7.1 & 0.94 & $<0.05$ \\
\hline $97 \mathrm{AZ}-4$ & 4.0 & 22. & 4.1 & 11. & 1.3 & 7.3 & 0.95 & $<0.05$ \\
\hline $97 \mathrm{AZ}-5$ & 4.8 & 27. & 4.8 & 13. & 1.6 & 8.8 & 1.1 & $<0.05$ \\
\hline $97 \mathrm{AZ}-6$ & 4.8 & 27. & 4.9 & 13. & 1.6 & 8.8 & 1.1 & $<0.05$ \\
\hline $97 \mathrm{AZ}-7$ & 4.8 & 27. & 4.8 & 13. & 1.6 & 8.7 & 1.1 & $<0.05$ \\
\hline $97 \mathrm{AZ}-8$ & 1.7 & 10. & 1.9 & 5.5 & 0.79 & 4.9 & 0.69 & $<0.05$ \\
\hline $97 \mathrm{AZ}-9$ & 2.2 & 13. & 2.4 & 6.8 & 1.0 & 6.3 & 0.91 & $<0.05$ \\
\hline $97 \mathrm{AZ}-10$ & 5.2 & 32. & 6.0 & 17. & 2.4 & 14. & 2.0 & $<0.05$ \\
\hline $97 \mathrm{AZ}-11$ & 3.4 & 21. & 4.1 & 13. & 1.8 & 12. & 1.8 & $<0.05$ \\
\hline $97 \mathrm{AZ}-12$ & $<0.05$ & $<0.05$ & $<0.05$ & $<0.05$ & $<0.05$ & $<0.05$ & $<0.05$ & $<0.05$ \\
\hline $97 \mathrm{AZ}-13$ & $<0.05$ & $<0.05$ & $<0.05$ & $<0.05$ & $<0.05$ & $<0.05$ & $<0.05$ & $<0.05$ \\
\hline $97 \mathrm{AZ}-14$ & 2.7 & 15. & 2.8 & 7.4 & 0.91 & 4.9 & 0.64 & $<0.05$ \\
\hline $97 \mathrm{AZ}-15$ & 4.9 & 27. & 4.9 & 13. & 1.6 & 8.7 & 1.2 & $<0.05$ \\
\hline $97 \mathrm{AZ}-16$ & 6.1 & 34. & 5.9 & 15. & 1.9 & 10. & 1.3 & $<0.05$ \\
\hline $97 \mathrm{AZ}-17$ & 6. & 35. & 6.2 & 16. & 2.0 & 11. & 1.3 & $<0.05$ \\
\hline
\end{tabular}


Table 7- Results of isotopic analyses of water samples. $\delta^{18} \mathrm{O}$ and $\delta \mathrm{D}$ are reported in permil deviation relative to standard mean ocean water (SMOW); $\delta^{34} \mathrm{~S}$ is reported in permil deviation from Cañon Diablo troilite (CDT).

\begin{tabular}{|l|c|c|c|c|}
\hline Sample No. & $\delta^{18} \mathrm{O}$ in $\mathrm{H}_{2} \mathrm{O}$ & $\delta \mathrm{D}$ in $\mathrm{H}_{2} \mathrm{O}$ & $\delta^{18} \mathrm{O}$ in $\mathrm{SO}_{4}$ & $\delta^{34} \mathrm{~S}^{\text {in }} \mathrm{SO}_{4}$ \\
\hline $97 \mathrm{AZ}-1$ & -8.5 & -59.4 & & \\
\hline $97 \mathrm{AZ}-2$ & -8.1 & -56.6 & 2.3 & -6.2 \\
\hline $97 \mathrm{AZ}-3$ & -8.9 & -62.2 & 1.3 & -5.2 \\
\hline $97 \mathrm{AZ}-4$ & -4.5 & -58.2 & -1.0 & -3.7 \\
\hline $97 \mathrm{AZ}-5$ & & -52 & -0.4 & -4.0 \\
\hline $97 \mathrm{AZ}-6$ & -6.92 & -50.4 & 0.0 & -3.5 \\
\hline $97 \mathrm{AZ}-7$ & & -49.7 & 0.4 & -3.5 \\
\hline $97 \mathrm{AZ}-8$ & & -55 & -0.4 & -3.3 \\
\hline $97 \mathrm{AZ}-9$ & & -64.2 & 0.3 & -3.3 \\
\hline $97 \mathrm{AZ}-10$ & -9.14 & -59.3 & 0.9 & -4.0 \\
\hline $97 \mathrm{AZ}-11$ & -8.83 & -64.6 & 1.2 & -6.9 \\
\hline $97 \mathrm{AZ}-12$ & & -73.8 & 1.7 & -1.3 \\
\hline $97 \mathrm{AZ}-13$ & -8.32 & -56.3 & 9.2 & -2.4 \\
\hline $97 \mathrm{AZ}-14$ & -7.53 & -54 & 0.5 & -2.8 \\
\hline $97 \mathrm{AZ}-15$ & -6.81 & -51.4 & -0.2 & -3.3 \\
\hline $97 \mathrm{AZ}-16$ & & -51.1 & -0.2 & -3.4 \\
\hline $97 \mathrm{AZ}-17$ & & -48.1 & 0.1 & -3.1 \\
\hline
\end{tabular}


Chemical character of the waters:

The general chemical character of the waters can be depicted by a number of methods, including Piper diagrams, Durov plots, etc. (cf. Freeze and Cherry, 1979; Domenico and Schwartz, 1998). The data from Tables 3 and 4 are shown in a Piper diagram in figure 3. As seen in the figure, the cation composition of the waters ranges from Ca-dominated through a mix of $\mathrm{Ca}-\mathrm{Mg}-\mathrm{Na}+\mathrm{K}$ dominated. The anion compositions, on the other hand, are nearly always sulfate dominated. The cation composition most likely varies as a result of the differences in the chemistry of the rocks with which the waters come into contact. The sulfate-dominated waters result from the oxidation of pyrite in the rocks, which produces sulfate.

Stable isotope data indicate that all of the sampled waters are of meteoric origin. The waters from Alum Gulch, downstream from the World's Fair mine drainage, are somewhat evaporated, as indicated by $\delta \mathrm{D}$ and $\delta^{18} \mathrm{O}$ enrichment. Sulfur isotope data for dissolved sulfate in mine drainage waters vary in $\delta^{34} \mathrm{~S}_{\mathrm{SO} 4}$ from -6.9 to $-2.8 \%$. These values are similar to $\delta^{34} \mathrm{~S}$ values of primary sulfides in nearby ore deposits (Shanks and Lichte, 1996). Oxygen isotopes values of sulfate in mine drainage waters have $\delta^{18} \mathrm{O}_{\mathrm{SO}}$ from -1.0 to $2.3 \%$, about 8$10 \%$ enriched relative to local meteoric water $\delta^{18} \mathrm{O}$. These values would indicate isotopic equilibrium exchange between $\mathrm{SO}_{4}$ and $\mathrm{H}_{2} \mathrm{O}$ at a temperature of about $200^{\circ} \mathrm{C}$ (Mizutani and Rafter, 1969), which is unrealistically high. More likely dissolved $\mathrm{SO}_{4}$ that forms as a result of bacterial sulfide mineral oxidation in mine tailing and working has incorporated some atmospheric oxygen, as demonstrated experimentally by Krouse et al. (1991).

\section{Conclusions}

Chemical analyses are presented for surface-water samples collected in 1997 in the Patagonia Mountains of southern Arizona. A full suite of inorganic chemical constituents is presented in this report. Data quality is assured by a variety of methods, which are oriented towards assuring accuracy and precision of the analytical results.

Typical of acid mine drainage waters, the samples reported on here have low $\mathrm{pH}$, and high concentrations of sulfate and metals. In most cases, sulfate constitutes $>95 \%$ of the total anion composition, but the cation character of the samples is more variable. In some cases, $\mathrm{pH}$ is sufficiently low that hydronium ion $\left(\mathrm{H}_{3} \mathrm{O}^{+}\right)$figures significantly into the anioncation charge balance.

Oxygen and hydrogen isotopes indicate that mine drainage exiting underground workings is meteoric water and becomes somewhat evaporated as it moves downstream significant distances in drainages. Sulfur and oxygen isotope values of sulfate in mine drainage waters indicate sulfur is derived from sulfides and some atmospheric oxygen may be incorporated. 


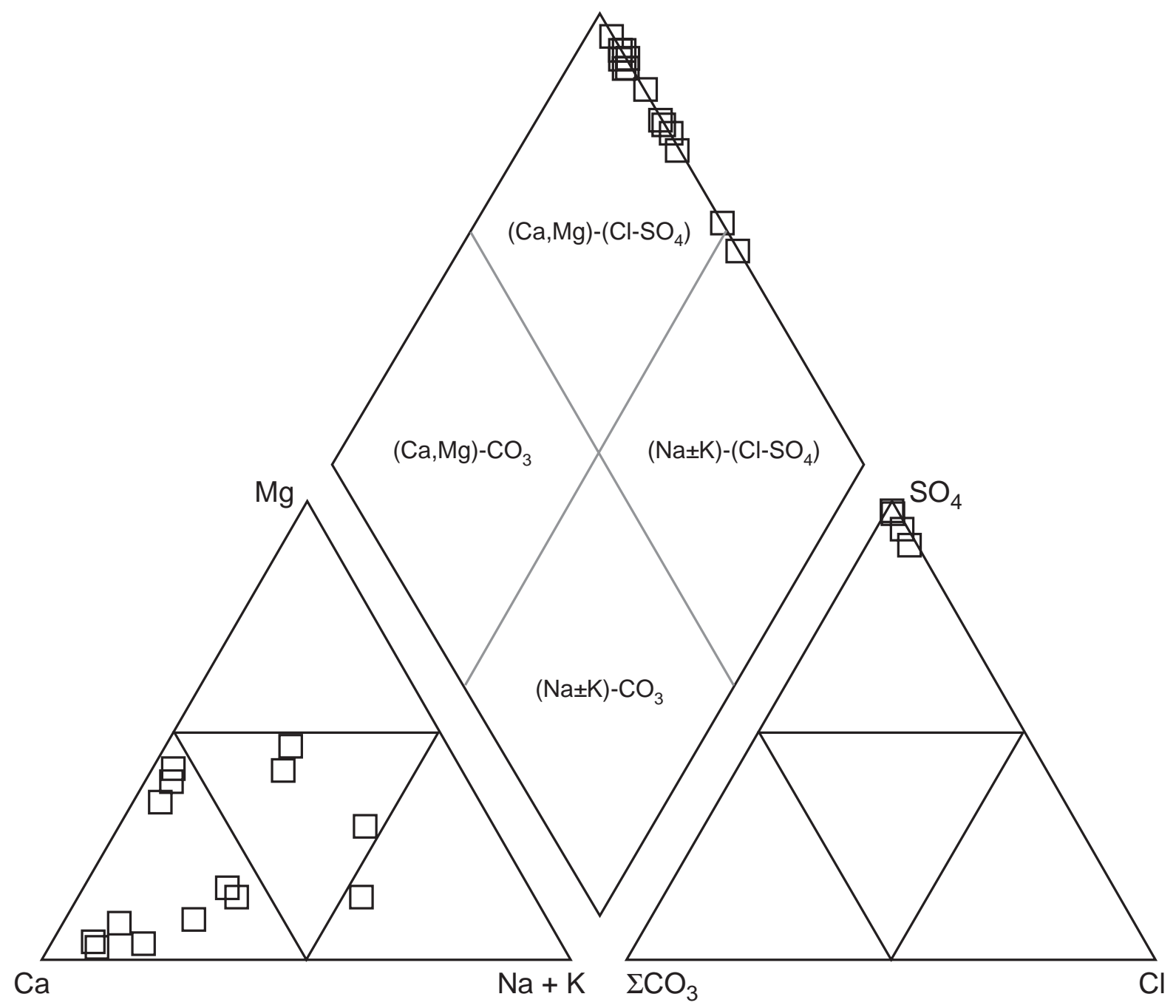

Figure 3- Piper plot for the water samples collected in the Patagonia Mountains study area. 


\section{References Cited}

Briggs, P.H. and Fey, D.L., 1996, Twenty-four elements in natural and acid mine waters by inductively coupled plasma-atomic emission spectrometry, in Arbogast, B.F., ed., Analytical methods manual for the Mineral Resource Program, U.S. Geological Survey: U.S. Geological Survey Open-File Report 96-525, p.95-101.

Clayton, R.N., and Mayeda, T.K., 1963, The use of bromine pentafluoride in the extraction of oxygen in oxides and silicates for isotopic analysis.: Geochim. Cosmochim. Acta, v. 27, p. 43-52.

Clesceri, L.S., Greenberg, A.E., Eaton, A.D., eds., 1998, Standard Methods for the Examination of Water and Wastewater (20th ed.): Washington, D.C., American Public Health Association.

Coleman, M.L., Shepherd, T.J., Durham, J.J., Rouse, J.E., and Moore, G.R., 1982, Reduction of water with zinc for hydrogen isotope analysis: Analytical Chemistry, v. 54, no. 6, p. 993-995.

Domenico, P.A., Schwartz, F.W., 1998, Physical and Chemical Hydrogeology (2nd ed.): New York, John Wiley and Sons, Inc., 506 p.

Ficklin, W.H., and Mosier, E.L., 1999, Field methods for sampling and analysis of environmental samples for unstable and selected stable constituents, in Plumlee, G.S., and Logsdon, M.J., The Environmental Geochemistry of Mineral Deposits: Reviews in Economic Geology, Volume 6A, Society of Economic Geologists, Littleton, Colorado, pp. 249-264.

Freeze, R.A., and Cherry, J.A., 1979, Groundwater: Englewood Cliffs, Prentice-Hall, Inc., $604 \mathrm{pp}$.

Krouse, H.R., Gould, W.D., McCready, R.G.L., and Rajan, S., 1991, (18)O incorporation into sulphate during the bacterial oxidation of sulphide minerals and the potential for oxygen isotope exchange between $\mathrm{O} 2, \mathrm{H} 2 \mathrm{O}$ and oxidized sulphur intermediates: Earth and Planetary Science Letters, v. 107, no. 1, p. 90-94.

Lamothe, P.J., Meier, A.L., and Wilson, S., 1999, The determination of forty-four elements in aqueous samples by inductively coupled plasma- mass spectrometry, U.S. Geological Survey Open-File Report 99-151, 14 p.

Mizutani, Y., and Rafter, T.A., 1969, Oxygen isotopic composition of sulphates; part 3, Oxygen isotopic fractionation in the bisulphate ion-water system: N.z. J. Sci., v. 12, no. 1, p. 54-59.

Schrader, F.C., 1915, Mineral deposits of the Santa Rita and Patagonia mountains, Arizona,, with contributions by J.M. Hill: U.S. Geological Survey Bulletin 582, 373 pp.

Shanks, W.C. III and Lichte, F.E., 1996, Trace element and stable isotope geochemistry of environmental minerals and acid rock drainage: Patagonia Mountains, Arizona: Geological Society of America, Annual Meeting, Abstracts with Program. 